\title{
Prevalencia y tipología de eventos estresantes en una muestra española con trastorno adaptativo: un estudio exploratorio
}

\author{
SANDRA ROJAS RODRÍGUEZ \\ al189941@uji.es \\ Daniel Campos Bacas \\ camposd@uji.es \\ MAR MolÉs AMPOSTA \\ molesm@uji.es \\ Soledad Quero Castellano \\ squero@uji.es
}

\section{Resumen}

Introducción: El trastorno adaptativo (TA) se produce como respuesta a eventos estresantes que ocasionan sintomatología emocional o comportamental clínicamente significativa. El término evento estresante abarca acontecimientos muy variados. Las investigaciones a este respecto son escasas, por lo que es necesario evaluar la representación de estos en población española. El objetivo de este trabajo es estudiar la prevalencia y la tipología de eventos estresantes en una muestra de pacientes con TA, así como explorar la existencia de diferencias entre los tipos de eventos estresantes y determinadas variables sociodemográficas y clínicas. Método: La muestra estaba compuesta por 91 pacientes con TA, con una edad media de 31,67 años (DT $=9,75$ ). Se incluyeron variables sociodemográficas, así como variables clínicas evaluadas antes del tratamiento (depresión, síntomas de estrés y pérdida y crecimiento postraumático). Resultados: Los resultados revelaron cuatro categorías de eventos: ruptura de pareja $(40,7 \%)$, problemática familiar $(29,7 \%)$, problemas laborales $(14,3 \%)$ y enfermedad (15,4\%). No se hallaron diferencias significativas entre los tipos de eventos estresantes en ninguna de las variables sociodemográficas ni clínicas, a excepción de las puntuaciones obtenidas en el Inventario de estrés y pérdida, siendo mayores en aquellas personas cuyo evento estresante fue una ruptura de pareja en comparación con la problemática familiar. Conclusiones: En este estudio se presentan, por primera vez, datos exploratorios sobre la prevalencia y tipología de eventos estresantes en una muestra clínica española con TA, así como su relación con variables sociodemográficas y clínicas. Es necesario continuar investigando para esclarecer el diagnóstico y proporcionar un adecuado tratamiento para los TA.

Palabras clave: trastorno adaptativo, tipología eventos estresantes, prevalencia, datos sociodemográficos. 


\section{Abstract}

Introduction: Adjustment Disorder (AD) occurs in response to stressful events and it causes clinically significant emotional or behavioural symptoms. The term stressful event covers a wide variety of events. Research in this regard is limited, so it is necessary to evaluate the distribution of these events in Spanish population. The aim of this work is to study the prevalence and types of stressful events in a sample of patients with $A D$ and explore whether there are differences between types of stressful events and some sociodemographic and clinical variables. Method: The sample consisted of 91 patients diagnosed with $A D$, with mean age of $31.67(S D=9.75)$. Sociodemographic variables and other clinical variables assessed before treatment (depression, stress and loss symptoms and posttraumatic growth) are included in this study. Results: The results revealed four types of events: relationship breakup $(40.7 \%)$, family problems (29.7\%), work problems (14.3\%) and disease (15.4\%). No significant differences between the types of stressful events in any of the sociodemographic and clinical variables were found, with the exception of the scores obtained for the inventory of stress and loss, being higher in those whose stressful event was a breakup, compared to family problems. Conclusions: This study presents, for the first time, exploratory data on the prevalence and type of stressful events in a Spanish clinical sample with $A D$, as well as their relationship with sociodemographic and clinical variables. More research is needed in order to clarify the diagnosis and to provide a suitable treatment for $A D$.

Keywords: Adjustment Disorder, stressful events typology, prevalence, sociodemographic data.

\section{Introducción}

A lo largo de nuestra vida nos enfrentamos a diversas situaciones estresantes que debemos afrontar, a la mayoría de las cuales conseguimos adaptarnos de modo apropiado. No obstante, en ocasiones algunas personas no poseen las estrategias de afrontamiento adecuadas, por lo que se generan en ellas síntomas emocionales y conductuales que repercuten de forma negativa en diversos ámbitos de sus vidas, dando lugar a lo que se conoce como trastorno adaptativo (TA).

Los TA se definen en el DSM-V (APA, 2013) como el desarrollo de sintomatología emocional o comportamental en respuesta a un factor o factores de estrés claramente identificables (criterio A). Esta sintomatología debe producirse en los tres meses siguientes al inicio del factor o factores estresantes (criterio A) y debe cesar dentro de los seis meses siguientes a la desaparición del evento estresante (criterio E). Así mismo, esta sintomatología resulta clínicamente significativa, bien provocando un malestar intenso desproporcionado a la gravedad o intensidad del factor de estrés (teniendo en cuenta el contexto externo y los factores culturales que podrían influir en la gravedad y la presentación de los síntomas), bien provocando un deterioro significativo en el ámbito social, laboral u otras áreas importantes del funcionamiento (criterio B). Solo se establecerá este diagnóstico si la persona no cumple los criterios exigidos para otros trastornos y siempre que no sea una exacerbación de otro trastorno mental persistente (criterio $\mathrm{C}$ ). Además, los síntomas no deben representar al duelo normal (criterio D). Una vez se diagnostica este trastorno, se requiere especificar el subtipo en función de los 
síntomas que predominan, pudiendo ser: con estado de ánimo deprimido, con ansiedad, con ansiedad mixta y estado de ánimo deprimido, con alteración de la conducta, con alteración mixta de las emociones o la conducta o sin especificar.

En cuanto a los datos de prevalencia de este trastorno, las cifras varían en función de la población estudiada y del método de evaluación utilizado, aunque ciertamente constituye un problema frecuente de salud (APA, 2013). Únicamente existe un gran estudio epidemiológico que ha incluido el TA como categoría diagnóstica en su investigación, aunque solo el subtipo con estado de ánimo depresivo, el ODIN (Outcomes of Depression International Network; Ayuso-Mateos y cols., 2001). Este estudio incluía cinco países europeos (Irlanda, Reino Unido, España, Noruega y Finlandia) y se encontró una prevalencia global de 0,3\%, aunque esta cifra variaba en función de los países. Por otra parte, la APA (2013) indica que entre el 5 y el $20 \%$ de las personas tratadas en régimen ambulatorio sufre este trastorno y, además, en el entorno hospitalario psiquiátrico, es de los trastornos más diagnosticados, alcanzando el $50 \%$ de los casos. Igualmente, su prevalencia es frecuente en personas que padecen condiciones médicas, especialmente si estas son graves (Strain y cols., 1998). Esta notable diferencia en cuanto a índices de prevalencia podría deberse, según Casey y cols. (2006), a los problemas de definición del TA y a la escasa sensibilidad de los instrumentos de evaluación existentes para este problema.

En cuanto a la relevancia de este trastorno, cabe señalar que algunos estudios indican que este se relaciona con elevadas tasas de autolesiones, entre un $25 \%$ y $60 \%$ de los casos, dependiendo de la edad (Pelkonen, Marttunen, Henriksson y Lonnqvist, 2005; Kryzhanovskaya y Canterbury, 2001). Además, se ha encontrado que las conductas suicidas se producen antes en el curso del TA que en la depresión mayor (Runeson, Beskow y Waem, 1996), así como el suicidio (Portzky, Audenaert y van Heeringen, 2005; Schnyder y Valach, 1997). Así mismo, Casey, Jabbar, O'Leary y Doherty (2015) hallaron que las personas con TA muestran tendencias suicidas a menores puntuaciones de gravedad de los síntomas que aquellos con depresión mayor, cuando ambos trastornos son de elevada gravedad, sugiriendo que el grupo con TA es más vulnerable a la tendencia suicida. Igualmente, es importante destacar la influencia del mismo en la salud pública, en lo que a términos económicos se refiere, pues es uno de los trastornos que mayor bajas laborales causa, por encima de otros trastornos como la depresión mayor o los trastornos de ansiedad (van der KlinK, Blonk, Schene y van Dijk, 2003).

Por otra parte, el término evento estresante hace referencia a una diversa variedad de sucesos, como, por ejemplo, problemas familiares, económicos, sociales, etc. (Robles, 2011). Así mismo, el suceso estresante puede ser un único evento o puede haber múltiples sucesos estresantes, puede ser recurrente o continuo, afectar a un solo individuo o a un grupo más grande y puede estar relacionado con el cambio vital de la persona (APA, 2013).

Cabe señalar que son escasos los estudios que han investigado la prevalencia de los eventos estresantes que suelen desencadenar el TA. Por ejemplo, en el estudio de Despland, Monod y Ferrero (1995) se encontró que los eventos estresantes más prevalentes eran los problemas conyugales (43\%), seguidos de los problemas familiares (17\%), los problemas laborales (14\%) y otros (26\%). Otro ejemplo en población española es el de Mur, Díez-Caballero, Florensa y Pérez (2005), quienes hallaron que los factores estresantes más habituales eran los factores laborales (30\%), familiares (25\%) y cambios de ciclo vital $(20 \%)$.

Por otra parte, en función de ciclo vital, Newcorn, Strain y Mezzich (2000) indican que los principales factores estresantes encontrados en la población adulta con TA son los problemas de pareja, separación, cambio de lugar de residencia y problemas económicos, mientras que en adolescentes serían los problemas académicos. En línea con lo anterior, Robles (2011) señala que en los adultos son más frecuentes los problemas laborales, económicos y de pareja, las situaciones de duelo, el desgaste profesional (burnout) y el acoso laboral (mobbing), 
mientras que en la adolescencia son más característicos los problemas académicos, la separación de los padres, el rechazo parental y las situaciones de acoso escolar (bullying) (Carson, Butcher y Mineka, 2000; Gómez-Macías y cols., 2006).

Con todo, es sorprendente el hecho de que el TA sea uno de los trastornos sobre los que menos se ha investigado y publicado desde que se identificó (Botella, Baños y Guillén, 2008). Por tanto, es necesario seguir investigando acerca de este trastorno, debido a la dificultad para establecer el diagnóstico y las grandes implicaciones que conlleva, como el elevado porcentaje de bajas laborales y el porcentaje de autolesiones y suicidios anteriormente mencionados. Así mismo, conocer aquellos eventos que han originado el trastorno, así como la posible influencia de los mismos en determinadas variables sociodemográficas y clínicas, nos ayudará a comprender mejor esta problemática con el fin de proporcionar un adecuado tratamiento a la misma.

De esta manera, el objetivo de este trabajo es estudiar la prevalencia y la tipología de eventos estresantes en una muestra de pacientes con TA, así como explorar la existencia de diferencias entre los tipos de eventos estresantes y determinadas variables sociodemográficas y clínicas.

\section{Método}

\section{Participantes}

En el estudio participaron 91 pacientes del Servicio de Asistencia Psicológica (SAP) de la Universitat Jaume I diagnosticados de TA. El 73,6 \% fueron mujeres y el 26,4 \% varones. La media de edad de los participantes fue de 31,67 (DT =9,76) con un rango de 18 a 54 años. Los subtipos diagnósticados fueron: con estado de ánimo depresivo (15,4\%), con ansiedad $(7,7 \%)$, mixto con estado de ánimo depresivo y ansiedad $(74,7 \%)$ y alteración mixta de las emociones y del comportamiento $(2,2 \%)$.

\section{Medidas}

En las sesiones de evaluación para delimitar el diagnóstico de TA se recogieron datos acerca de los eventos estresantes que habían originado el trastorno, además de diversas variables sociodemográficas como el sexo, la edad y el nivel educativo.

A continuación se describen los instrumentos utilizados en el presente estudio para evaluar las variables clínicas:

Inventario de depresión de Beck (BDI-II: Beck Depression Inventory-Second Edition; Beck, Steer y Brown, 1996. Validado en población española por Sanz, Vázquez y Perdigón, 2003). Este instrumento evalúa la sintomatología depresiva. Se trata de un autoinforme de 21 ítems que se contestan en una escala tipo Likert de 0 (totalmente desacuerdo) a 3 (totalmente de acuerdo), en el que las puntuaciones finales indican: de 0 a 9 , no sintomatología depresiva; de 10 a 18, síntomatologia leve a moderada; de 19 a 29, sintomatologia moderada a grave y, por último, de 30 a 63 sintomatologia grave. La validación española ha mostrado tener buenas propiedades psicométricas (Sanz y cols., 2003).

Inventario de estrés y pérdida (IEP; Quero, Molés, Mor, Baños y Botella, 2014; adaptado de Prigerson y cols., 1995). Este instrumento evalúa la intensidad con la que interfiere la situación o la persona perdida en la vida de la persona. Se trata de un autoinforme de 17 ítems que se contestan en una escala Likert de 0 (nunca) a 4 (siempre), siendo la puntuación mínima 0 y la máxima 68. Datos preliminares de la validación mostraron excelentes coeficientes de fia- 
bilidad test-restest $(0,90)$ y de consistencia interna en población española tanto general $(0,91)$ como clínica con TA $(0,86)$ (Quero y cols., 2014).

Inventario de crecimiento postraumático (PTGI: Post Traumatic Growth Inventory; Tedeschi y Calhoun, 1996). Este instrumento evalúa los posibles cambios positivos que pueden experimentar las personas tras haber sufrido un acontecimiento traumático o adverso. Se trata de un autoinforme de 21 ítems que se contestan en una escala Likert de 0 («No experimenté este cambio como resultado de la crisis») a 5 («Experimenté este cambio en medida muy grande como resultado de la crisis»), siendo la puntuación mínima 0 y la máxima 105. Puntuaciones elevadas en este inventario indican un mayor grado de crecimiento postraumático percibido. Se han obtenido elevados índices de consistencia interna tanto para las 5 escalas como para la puntuación total (Ho, Chu y Yiu, 2008; Tedeschi y Calhoun, 1996). Datos preliminares de la validación de este cuestionario en muestra española diagnosticada de TA $(\mathrm{N}=101)$ mostraron excelentes coeficientes de consistencia interna (alfa de Cronbach 0,90) y fialibilidad test-retest $(0,95)$ para la puntuación total del cuestionario (Rachyla, Reyes-Torres, Molés y Quero, 2016).

\section{Procedimiento}

Se recogieron datos de pacientes del SAP diagnosticados de TA, referidos a las variables sociodemográficas y clínicas anteriores al tratamiento, correspondientes a las sesiones de evaluación inicial. Seguidamente, se procedió a la realización de los análisis correspondientes mediante el Statistics Package for Social Sciences (SPSS-22), versión en español. Todos los participantes formaron parte de un estudio de investigación donde posteriormente recibieron tratamiento psicológico para el TA y firmaron el consentimiento informado participando de forma voluntaria.

\section{Resultados}

En primer lugar, por lo que respecta a la tipología de los eventos estresantes, los pacientes se agruparon en cuatro grandes categorias: ruptura de pareja (incluye ruptura sentimental, divorcio, separación y problemas derivados), problemática familiar (incluye problemas con algún miembro de la familia y problemas económicos), problemas laborales (incluye problemas en el trabajo, despido, mobbing y problemas con los estudios) y enfermedad (incluye enfermedad propia o accidente y enfermedad en algún miembro de la familia). Los porcentajes hallados para cada categoría se presentan en la figura 1.

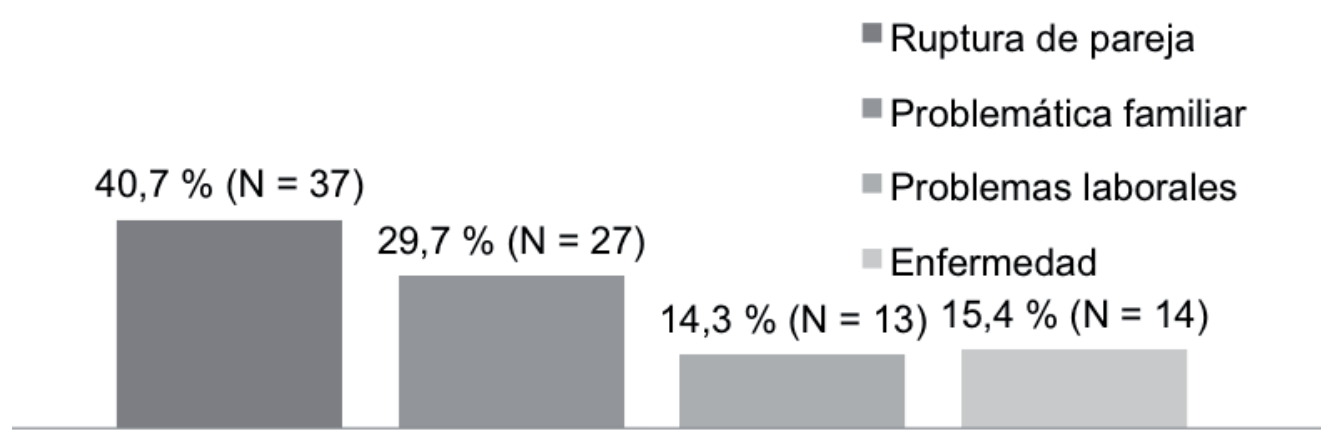

Figura 1. Prevalencia de la tipología de eventos estresantes 
En segundo lugar, se analizaron las diferencias entre las categorias de eventos estresantes y determinadas variables sociodemográficas y clínicas. Para ello se realizó una prueba de Chi-cuadrado $\left(\mathrm{X}^{2}\right)$ en aquellas variables cualitativas como el sexo y el nivel educativo (véase la tabla 1) y un ANOVA para las variables cuantitativas, como son la edad, las puntuaciones en el BDI-II, IEP y PTGI (véase la tabla 2). Los resultados no mostraron diferencias estadísticamente significativas entre tipos de eventos estresantes en ninguna variable, a excepción de las puntuaciones obtenidas en el IEP $(F=3,460 ; p<0,05)$. Las pruebas post-hoc indicaron que aquellas personas cuyo evento estresante fue una ruptura de pareja obtuvieron puntuaciones mayores de estrés y pérdida en comparación con la problemática familiar.

Tabla 1

Diferencias entre las categorias de eventos estresantes en función de las variables sexo y nivel educativo

\begin{tabular}{llcccc}
\hline & & \multicolumn{2}{c}{$f$} & $X^{2}$ & $p$ \\
\hline \multirow{5}{*}{ Sexo } & Rombres & Mujeres & & \\
& Pb. familiar & 5 & 28 & & \\
& Pb. laboral & 6 & 22 & 3,592 & 0,309 \\
& Enfermedad & 4 & 7 & & \\
\hline \multirow{5}{*}{ Nivel educativo } & Prim./Secun. & Universitarios & & \\
& Ruptura & 8 & 29 & & \\
& Pb. familiar & 8 & 10 & 2,625 & \\
& Pb. laboral & 5 & 8 & & \\
& Enfermedad & 2 & 12 & & \\
\hline
\end{tabular}

Tabla 2

Diferencias entre las categorías de eventos estresentes en función de la edad y puntuaciones obtenidas en BDI-II, IEP Y PTGI

\begin{tabular}{|c|c|c|c|c|c|}
\hline & & M & D.T. & $\mathrm{F}$ & $\mathrm{p}$ \\
\hline \multirow{4}{*}{ Edad } & Ruptura & 30,86 & 9,461 & \multirow{4}{*}{0,552} & \multirow{4}{*}{0,668} \\
\hline & $\mathrm{Pb}$. familiar & 31,41 & 9,974 & & \\
\hline & $\mathrm{Pb}$. laboral & 34,77 & 10,059 & & \\
\hline & Enfermedad & 31,43 & 10,338 & & \\
\hline \multirow{4}{*}{ BDI-II } & Ruptura & 25,50 & 10,796 & \multirow{4}{*}{2,196} & \multirow{4}{*}{0,094} \\
\hline & $\mathrm{Pb}$. familiar & 20,37 & 9,584 & & \\
\hline & $\mathrm{Pb}$. laboral & 25,46 & 6,851 & & \\
\hline & Enfermedad & 19,64 & 10,485 & & \\
\hline
\end{tabular}




\begin{tabular}{llcccc}
\hline & & M & D.T. & F & p \\
\hline \multirow{4}{*}{ IEP } & Ruptura & 40,457 & 10,514 & & \\
& Pb. familiar & 30,957 & 11,83 & \multirow{2}{*}{0,460} & 0,02 \\
& Pb. laboral & 31,167 & 8,233 & & \\
& Enfermedad & 35,786 & 18,419 & & \\
\hline \multirow{3}{*}{ PTGI } & Ruptura & 34,222 & 19,372 & & 0,405 \\
& Pb. familiar & 40,815 & 19,464 & & \\
& Pb. laboral & 30,846 & 19,261 & & \\
\hline
\end{tabular}

\section{Discusión y conclusiones}

Los resultados obtenidos en este trabajo muestran datos exploratorios sobre la prevalencia y la tipología de eventos estresantes en una muestra clínica española con TA, así como su relación con variables sociodemográficas y clínicas.

En cuanto a la tipología de los eventos estresantes, se hallaron cuatro grandes categorias de eventos relacionados con: ruptura de pareja (ruptura sentimental, divorcio, separación y problemas derivados), problemática familiar (problemas con algún miembro de la familia y problemas económicos), problemas laborales (problemas en el trabajo, despido, mobbing y problemas con los estudios) y enfermedad (enfermedad propia o accidente y enfermedad familiar). Los porcentajes hallados en el presente estudio de prevalencia de las distintas categorías son semejantes a los obtenidos en el estudio de Despland y cols. (1995), quienes hallaron que la problemática más prevalente era la relacionada con los problemas maritales con un $43 \%$ de los casos (siendo en nuestro estudio de $40,7 \%$, en el caso de ruptura de pareja), seguida de un $17 \%$ en el caso de los problemas familiares (29,7\% en nuestro estudio) y de un $14 \%$ en el caso de los problemas ocupacionales (14,3\% en nuestro estudio). No obstante, en el caso del estudio de Mur y cols. (2005), llevado a cabo en población española, nuestros resultados son diferentes por lo que se refiere a la problemática laboral, hallando estos autores un porcentaje mayor (30 \%) que el obtenido en nuestro estudio (14,3\%), mientras que el porcentaje encontrado para los problemas familiares $(25 \%)$ fue más similiar a los resultados hallados en el presente estudio $(29,7 \%)$. Por otra parte, teniendo en cuenta el ciclo vital, nuestros datos son similares con los obtenidos en estudios previos como el de Newcorn y cols. (2000), quienes mencionan que los factores estresantes más habituales en el TA en adultos son los problemas de pareja, separación, cambio de lugar de residencia y problemas económicos. De la misma manera, nuestros datos son semejantes a aquellos hallados en la literatura por Robles (2011) quien señala que los problemas estresantes más frecuentes en los adultos se relacionan con los problemas laborales, económicos, de pareja, situaciones de duelo, el burnout y el mobbing (Carson y cols., 2000; Gómez-Macías y cols., 2006).

Respecto a la diferencias entre las categorías de eventos estresantes en función de variables sociodemográficas y clínicas, únicamente se encontraron diferencias estadísticamente significativas en el caso de la puntuación obtenieda en el IEP, siendo mayor en aquellas personas cuyo evento estresante había sido una ruptura de pareja en comparación con quienes habían sufrido un problema familiar. Que nosotros sepamos, no existe literatura relacionada con las diferencias entre estos eventos en función de las variables estudiadas, por lo que sería conveniente continuar con su estudio en el futuro. 
Con todo, podemos concluir que, según nuestros resultados, el acontecimiento estresante más prevalente que daba lugar a un TA en esta muestra clínica fue haber sufrido una ruptura sentimental. Además, parece que sufrir este tipo de acontecimiento provoca una mayor sintomatología de estrés y pérdida.

Algunas limitaciones de nuestro estudio podrían estar relacionadas con las características de la muestra, ya que en nuestro caso podría existir un sesgo al tratarse de personas que acuden al SAP de la universidad, debido a que los pacientes que suelen acudir son personas jóvenes con estudios universitarios, lo cual podría explicar en parte las diferencias halladas con otros estudios. Así mismo, cabe señalar que se trata de un estudio exploratorio, por lo que los resultados hacen referencia a la muestran estudiada. Por otra parte, no se ha tenido en cuenta la implicación y la posible influencia de la tipología del estresor en los resultados de la terapia. En un futuro, se requieren estudios que aborden estos aspectos contando, al mismo tiempo, con un una mayor muestra que permita la generalización de los hallazgos.

En conclusión, es importante continuar estudiando esta problemática debido a que los estudios acerca del TA son escasos, a pesar de ser uno de los trastornos más frecuentes en los contextos de atención primaria y hospitalaria (Carta, Balestrieri, Murru y Carolina-Hardoy, 2009). Por ello, es necesario continuar investigando acerca de la prevalencia de los eventos estresantes y la posible existencia de diferencias entre los mismos, lo cual ayudaría a mejorar el diagnóstico $y$, de esta manera, proporcionar un adecuado tratamiento a quienes padecen un TA.

\section{Referencias bibliográficas}

American Psychiatric Association (2013). Diagnostic and statistical manual of mental disorders (5th ed.). Arlington, VA: American Psychiatric Publishing.

Ayuso-Mateos, J. L., Vázquez-Barquero, J. L., Dowrick, C., Lehtinen, V., Dalgard, O. S., Casey, P. y cols. (2001). Depressive disorders in Europe: prevalence figures from the ODIN study. The British Journal of Psychiatry, 179, 308-316.

Beck, A. T., Steer, R. A. y Brown, G. K. (1996). Manual for the Beck Depression Inventory-II. San Antonio, TX: Psychological Corp.

Botella, C., Baños, R. M. y Guillén, V. (2008). Una propuesta de tratamiento para los trastornos adaptativos: creciendo en la diversidad. En C. Vázquez y G. Hervás (eds.), Psicología positiva aplicada (pp.129-154). Bilbao: Desclée de Brouwer.

Carson, R. C., Butcher, J. N. y Mineka, S. (2000). Abnormal psychology and modern life. Boston: Allyn and Bacon.

Carta, M. G., Balestrieri, M., Murru, A. y Carolina-Hardoy, M. C., 2009. Adjustment Disorder: epidemiology, diagnosis and treatment. Clinical Practice \& Epidemiology in Mental Health, $5,1-15$.

Casey, P., Jabbar, F., O'Leary, E. y Doherty, A. M. (2015). Suicidal behaviours in adjustment disorder and depressive episode. Journal of affective disorders, 174, 441-446.

Casey, P., Maracy, M., Kelly, B. D., Lehtinen, V., Ayuso-Mateos, J. L., Dalgard, O. S. y cols. (2006). Can adjustment disorder and depressive episode be distinguished? Results from ODIN. Journal of Affective Disorders, 92, 291-297.

Despland J. N., Monod L. y Ferrero F. (1995). Clinical relevance of adjustment disorder in DSMIII-R and DSM-IV. Comprehensive Psychiatry, 36, 454-60.

Gómez-Macías, V., De Cos, A., García, C., García, F., Ballesteros, M. y Jiménez, M. C. (2006). Trastornos adaptativos: factores estresantes en el momento actual. Interpsiquis.

Ho, S. M., Chu, K. W. y Yiu, J. (2008). The relationship between explanatory style and post traumatic growth after bereavement in a non-clinical sample. Death Studies, 32, 461-478. 
Kryzhananovskaya, L. y Canterbury, R. (2001). Suicidal behaviour in patients with adjustment disorders. Crisis, 22, 125-131.

Mur, T., Díez-Caballero, M., Florensa, E. y Pérez, L. (2005). Descriptive study of adaptive disorders in a primary care centre. Atencion primaria / Sociedad Española de Medicina de Familia y Comunitaria, 36, 404.

Newcorn, J. H., Strain, J. J. y Mezzich, J. E. (2000). Adjustment disorders. En B. J. Sadock y V. A. Sadock (eds.), Comprehensive Textbook of Psychiatry. 7th ed. (pp. 1714-1722). Philadelphia: Lippincott Williams \& Wilkins.

Pelkonen, M., Marttunen, M., Henriksson, M. y Lonnqvist, J. (2005). Suicidality in adjustment disorder-clinical characteristics of adolescent outpatients. European Child \& Adolescent Psychiatry, 14, 174-180.

Portzky, G., Audenaert, K. y van Heeringen, K. (2005). Adjustment disorder and the course of the suicidal process in adolescents. Journal of Affective Disorders, 87, 265-270.

Prigerson, H. G., Maciejewsku, P. K., Reynolds, C. F., Bierhals, A. J., Newsom, J. T., Fasiczka, A. y cols. (1995). Inventory of Complicated Grief: A scale to measure maladaptive symptoms of loss. Psychiatry Research, 59, 65-79.

Quero, S., Molés, M., Mor, S., Baños, R. y Botella, C. (2014). Spanish Validation of the Complicated Grief Inventory adapted for Adjustment Disorders. Póster presentado en Association for Behavioral and Cognitive Therapies 48th Annual Convention, Philadelphia, United States.

Rachyla, I., Reyes-Torres, F., Molés, M. y Quero, S. (2016). Validación española del Inventario de Crecimiento Postraumático en pacientes con trastorno adaptativo: Análisis factorial exploratorio. Póster presentado en II Jornades d'Investigació per als Estudiants de la Facultat de Ciències de la Salut, Universitat Jaume I, Castellón.

Robles, H. (2011). Trastornos adaptativos. En V. E. Caballo, I. C. Salazar y J. A. Carrobles (eds.), Manual de psicopatología y trastornos psicológicos (pp. 707-727). Madrid: Pirámide.

Runeson, B. S., Beskow, J. y Waem, M., (1996). The suicidal process in suicides among young people. Acta Psychiatrica Scandinavica, 93, 35-42.

Sanz, J., Vázquez, C. y Perdigón, A. L. (2003). Adaptación española del Inventario para la Depresión de Beck-II (BDI-II): 2. Propiedades psicométricas en población general. Clínica y Salud: Revista de psicología clínica y salud, 14, 249-280.

Schnyder, U. y Valach, L. (1997). Suicide attempters in the emergency room population. General Hospital Psychiatry, 19,119-129.

Strain, J. J., Smith, G. C., Hammer, J. S., McKenzie, D. P., Blumenfield, M., Muskin, P. y cols. (1998). Adjustment disorder: a multisite study of its utilization and interventions in the consultation-liaison psychiatry setting. General Hospital Psychiatry, 20, 139-149.

Tedeschi, R. G. y Calhoun, L. G. (1996). The Posttraumatic Growth Inventory: Measuring the Positive Legacy of Trauma. Journal of Traumatic Stress, 9, 455-471.

van der Klink, J. L. J., Blonk, R. W. B., Schene, A. H. y van Dijk, F. J. H. (2003). Reducing long term sickness absence by an activating intervention in adjustment disorders: a cluster randomised controlled design. Occupational and Environmental Medicine, 60, 429-437.

Herbert, B. M. y Pollatos, O. (2012). The body in the mind: on the relationship between interoception and embodiment. Topics in Cognitive Science, 4, 692-704.

Jay, D. (1991). Effect of a dance program on the creativity of preschool handicapped children. Adapted physical activity quarterly, 8, 305-316.

Koch, S. C. (2006). Embodiment and creative arts therapy: From phenomenology to cognitive science. En S. Scoble (ed.), European Arts Therapy. Grounding the vision to advance theory and practice. Plymouth: University Plymouth Press. 
Kvale, S. (2011). Las entrevistas en investigación cualitativa. Madrid: Morata.

Lima de Figueiredo Queirós, M. M., Fernández Berrocal, P., Extremera, N. y Susana Queirós, P. (2006). Perceived emotional intelligence, response style and physical activity in old age. Ansiedad y Estrés, 12, 293-303.

Lobo, Y. B. y Winsler, A. (2006). The effects of a creative dance and movement program on the social competence of head start preschoolers. Social Development, 15(3), 501-519.

López, C. y Rueda, B. (2013). Siento, me muevo, luego existo. Revista de Educación, Motricidad e Investigación, 1, 14-29.

Mac Donald, C. J. (1991). Creative dance in elementary schools: A theoretical and practical justification. Canadian Journal of Education, 16, 434-441.

Maslow (1972). El Hombre autorrealizado. Barcelona: Kairos.

Payne, H. (1990). Creative movement and dance in groupwork. United Kingdom: Speechmark.

Peña, N. y Jaramillo, L. (1998). La danza, factor de promoción ético moral en los adolescentes marginados. Apunts: Educación física y deportes, 4(54), 12-21. Recuperado de: http:// www.raco.cat/index.php/ApuntsEFD/article/view/307339

Ratey, J. (2001). A User's Guide to the Brain. New York, NY: Random House Inc.

Rizzolatti, G. y Sinigaglia, C. (2006). So quel che fai. Il cervello che agisce e i neurona specchio. Milano: Raffaello Cortina Editore.

Rodríguez, R., Caja, M. y Gracia, P. (2013). Inteligencia Emocional y Comunicación: la conciencia corporal como recurso. Revista de Docencia Universitaria, 11, 213-241.

Salas-Calderón, S. (2007). Psicoterapia del movimiento : herramienta terapéutica con pacientes esquizofrénicos. Actualidades en Psicología, 21, 97-115.

Sebiani, L. (2005). Uso de la danza-terapia en la adaptación psicológica a enfermedades crónicas. Reflexiones, 84, 49-56.

Sheets-Johnstone, M. (2010). Why is movement therapeutic? American Journal of Dance Therapy, 32, 2-15. 